\title{
Combined Baerveldt glaucoma drainage implant surgery and surgical bleb revision for preventing a postoperative hypertensive phase
}

This article was published in the following Dove Press journal:

Clinical Ophthalmology

16 April 2014

Number of times this article has been viewed

\section{Motofumi Kawai \\ Toru Yamaguchi \\ Seigo Nakabayashi \\ Akitoshi Yoshida}

Department of Ophthalmology, Asahikawa Medical University, Asahikawa, Japan
Correspondence: Motofumi Kawai Department of Ophthalmology, Asahikawa Medical University, Midorigaokahigashi 2-I-I-I, Asahikawa, 078-8510, Japan

$\mathrm{Tel}+8 \mathrm{I} 166682543$

Fax +8I I66 682549

Email m-kawai@asahikawa-med.ac.jp
Background: In patients with severe optic nerve damage, it is crucial to prevent the hypertensive phase that can develop after Baerveldt glaucoma drainage implant (BGI) surgery. We describe the combination of BGI surgery with surgical bleb revision to prevent the postoperative hypertensive phase.

Case reports: We report two patients who underwent BGI surgery combined with surgical bleb revision. The combined surgery was performed in a 62-year-old man with open-angle glaucoma (patient 1) and a 37-year-old man with neovascular glaucoma (patient 2) at Asahikawa Medical University in 2013. Each patient had undergone a previous failed trabeculectomy and another trabeculectomy was expected to be unsuccessful. In patient 1 , the early postoperative intraocular pressure (IOP) was well controlled due to a well-functioning bleb, despite development of a small amount of hyphema. In contrast, in patient 2 the postoperative IOP was higher with poor bleb formation; however, after bleb needling, the IOP was well controlled. Thus, the usual techniques used after trabeculectomy were also applicable in this combined surgery. For comparison, we also report the case of a 54-year-old woman with secondary glaucoma who underwent BGI surgery alone (patient 3 ). The postoperative IOP remained relatively high compared to patients 1 and 2 .

Conclusion: Combining BGI surgery with surgical bleb revision might be effective to prevent the hypertensive phase that can occur after BGI surgery.

Keywords: Baerveldt glaucoma drainage implant, surgical bleb revision, intraocular pressure

\section{Introduction}

Transient intraocular pressure (IOP) elevation in the early postoperative period (ie, the hypertensive phase) can occur after Baerveldt glaucoma drainage implant (BGI) surgery. This complication occurs due to the temporary occlusion of the tube lumen, which completely inhibits aqueous drainage to the plate. Because a nonvalved BGI cannot adjust the amount of aqueous that drains (in contrast to other devices such as the Ahmed [New World Medical, Rancho Cucamonga, CA, USA] glaucoma valve), occluding the tube tightly with an absorbable ligature before inserting it into the anterior chamber ${ }^{1}$ is important to avoid overfiltration and subsequent hypotony, which are other major potential complications of BGI surgery. ${ }^{2}$

In Japan, most patients who undergo BGI surgery already have severe optic nerve damage due to prolonged glaucoma, because the surgery is currently limited to a subset of patients with refractory glaucoma, eg, patients who previously underwent repeated ocular surgeries, and those with no good prospect for a successful future 
trabeculectomy. ${ }^{3}$ Every effort should be made to avoid the hypertensive phase in these patients.

We describe combined BGI surgery and surgical bleb revision in two cases. Our results suggest that the hypertensive phase that follows BGI surgery can be prevented by combining BGI surgery with surgical bleb revision.

\section{Case reports}

The patients' characteristics are summarized in Table 1. The fundus photographs obtained from patients 1 and 2 are shown in Figure 1.

Patient 1, a 62-year-old man with open-angle glaucoma had undergone repeated trabeculectomies in the superior quadrants and had an IOP of $42 \mathrm{mmHg}$ in his left eye. The best-corrected visual acuity (BCVA) was 20/40. BGI surgery was performed in the inferior quadrant where the conjunctiva was intact. Because the optic nerve damage was severe due to the prolonged duration ( $>2$ years) of uncontrolled glaucoma, we performed BGI surgery in combination with surgical bleb revision for early IOP control.

After anesthesia was induced with a peribulbar injection of 2\% lidocaine, a clear corneal 6-0 polyglycolic acid (PGA) absorbable violet braided suture (Mani Inc, Tochigi, Japan) was placed in the peripheral cornea, and the area of the failed bleb in the superonasal quadrant was exposed (Figure 2A). The scarred conjunctiva was dissected posteriorly using blunt-tip scissors. A bare scleral flap was dissected using an ophthalmic knife until the aqueous humor egressed (Figure 2B). The fornix-based conjunctival flap was reapproximated to the limbus and closed with 10-0 nylon sutures at each end of the periotomy (Figure 2C).

A subconjunctival space in the inferotemporal quadrant was then created, and a $350 \mathrm{~mm}^{2}$ Baerveldt implant (Abbott Medical Optics Inc., Santa Ana, CA, USA) was positioned under the inferior and temporal rectus muscles (Figure 2D).
To minimize postoperative hypotony, the tube was tightly occluded by ligation with 8-0 PGA absorbable violet braided suture (Mani Inc) before insertion into the anterior chamber. Balanced salt solution was injected into the tube using a 27 gauge cannula to confirm that the tube was totally occluded. The plate was sutured to the sclera with 9-0 nylon sutures. The tube was then cut bevel up, and inserted into the anterior chamber via a needle track created with a 23 gauge needle (Figure 2E). To achieve early IOP control, a needle was used to penetrate both sides of the tube (ie, tube fenestrations were created) ${ }^{4}$ in three places (Figure $2 \mathrm{~F}$ ). A scleral graft was used to cover the outer portion of the tube (Figure 2G). The conjunctiva was sutured back in its original position using 8-0 silk sutures. Bleb formation was observed after irrigation with balanced salt solution through the paracentesis (Figure 2H). Subconjunctival injection of betamethasone and tobramycin was administered at the end of the surgery.

Although a small amount of hyphema developed, probably in relation to the surgical bleb revision, it resolved within 7 days postoperatively and did not affect bleb formation.

Patient 2 was a 37-year-old man with neovascular glaucoma caused by central retinal vein occlusion. The IOP in his left eye increased markedly to $68 \mathrm{mmHg}$ despite an intravitreal injection of bevacizumab (Avastin; Genentech, Inc., South San Francisco, CA, USA) and subsequent panretinal photocoagulation. The BCVA was $<20 / 200$. An initial trabeculectomy was performed; however, the bleb failed despite repeated needling. Due to extensive conjunctival scarring and severe iris neovascularization, which is likely to cause hyphema after trabeculectomy, BGI surgery was performed. To avoid the hypertensive phase, the BGI procedure was combined with surgical bleb revision. The surgical procedure was the same as that described for patient 1 .

Table I Patient characteristics

\begin{tabular}{|c|c|c|c|c|c|}
\hline Patient & Age (years) & Sex & Type of glaucoma (etiology) & Previous ocular surgery (date) & Surgical procedure (date) \\
\hline 1 & 62 & Male & $\begin{array}{l}\text { Open-angle glaucoma } \\
\text { (unknown) }\end{array}$ & $\begin{array}{l}\text { Trabeculectomy with MMC } \\
\text { (December 6, 20I0) } \\
\text { Phaco-trabeculectomy with MMC } \\
\text { (January } 26,201 \mathrm{I} \text { ) }\end{array}$ & $\begin{array}{l}\text { BGI combined with surgical } \\
\text { bleb revision (April 16, 20I3) }\end{array}$ \\
\hline 2 & 37 & Male & $\begin{array}{l}\text { Neovascular glaucoma } \\
\text { (CRVO) }\end{array}$ & $\begin{array}{l}\text { Intravitreal bevacizumab injection } \\
\text { (July } 22,20 \mathrm{II} \text { ) } \\
\text { Trabeculectomy with MMC } \\
\text { (June } 30,2012 \text { ) }\end{array}$ & $\begin{array}{l}\text { BGI combined with surgical } \\
\text { bleb revision (February 5, 20I3) }\end{array}$ \\
\hline 3 & 54 & Female & $\begin{array}{l}\text { Secondary glaucoma } \\
\text { (ocular sarcoidosis) }\end{array}$ & $\begin{array}{l}\text { Trabeculectomy with MMC } \\
\text { (February 6, 2007) } \\
\text { Trabeculectomy with MMC } \\
\text { (June 2, 2008) }\end{array}$ & BGI alone (November 30, 20I2) \\
\hline
\end{tabular}

Abbreviations: MMC, mitomycin C; BGI, Baerveldt glaucoma drainage implant; CRVO, central retinal vein occlusion. 


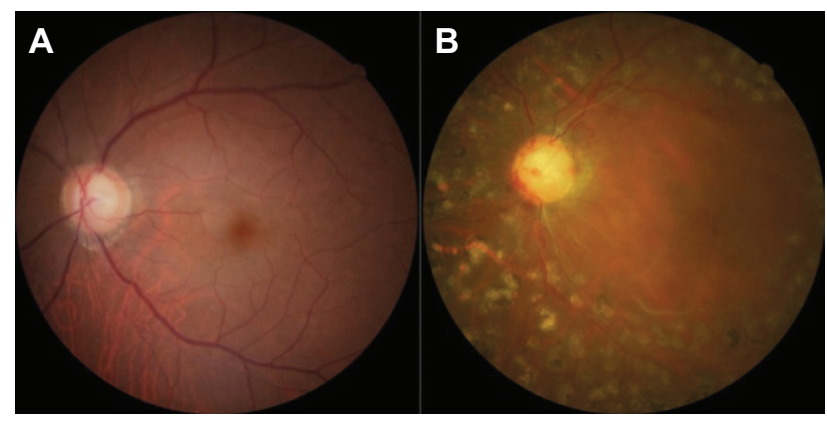

Figure I Fundus photographs of patient I (A) and patient 2 (B).

Notes: Severe optic nerve damage is observed in both patients. The cup-to-disc ratios are 0.8 to 0.9 and 0.9 in patients I and 2, respectively. In patient 2, scars from panretinal photocoagulation and closure of retinal vessels also are observed.

For comparison, we present a representative case treated with BGI surgery alone. Patient 3 was a 54-year-old woman with secondary glaucoma caused by ocular sarcoidosis. The IOP increased to $30 \mathrm{mmHg}$ in her right eye. The BCVA was $20 / 25$. She had previously undergone repeated trabeculectomies. Because of the severity of the conjunctival scarring in the superior quadrants, BGI surgery was performed in the inferior quadrant.

No signs of tubal obstruction were observed in any of the patients postoperatively. The changes in the postoperative IOP levels of the three patients are shown in Figure 3. The postoperative IOP was controlled by postoperative bleb management in patients 1 and 2 . In patient 3 , the postoperative IOP remained relatively high compared to patients 1 and 2 .

\section{Discussion}

We described BGI surgery combined with surgical bleb revision. The hypertensive phase was prevented postoperatively in our patients. Surgical bleb revision may also prevent the hypertensive phase due to tubal obstruction caused by fibrin, blood, or the iris. In a recent tube versus trabeculectomy study, ${ }^{5}$ the plate was positioned in the superotemporal quadrant, whereas we placed it in the inferior quadrant because the superior conjunctiva was cicatrized due to previous glaucoma surgeries. Because the surgical sites of each procedure do not overlap, the combined surgery can be adapted to any case.

One study reported that a hypertensive phase developed postoperatively in $82 \%$ of patients who were implanted with an Ahmed valve. ${ }^{6}$ Although combining tube implantation with a trabeculectomy might prevent the hypertensive phase, ${ }^{7}$ it was difficult to perform further trabeculectomies in our patients because the conjunctiva in the upper quadrants was cicatrized. If favorable outcomes of future trabeculectomies are expected, BGI surgery is not required. Although it was reported that transient IOP elevations after BGI surgery

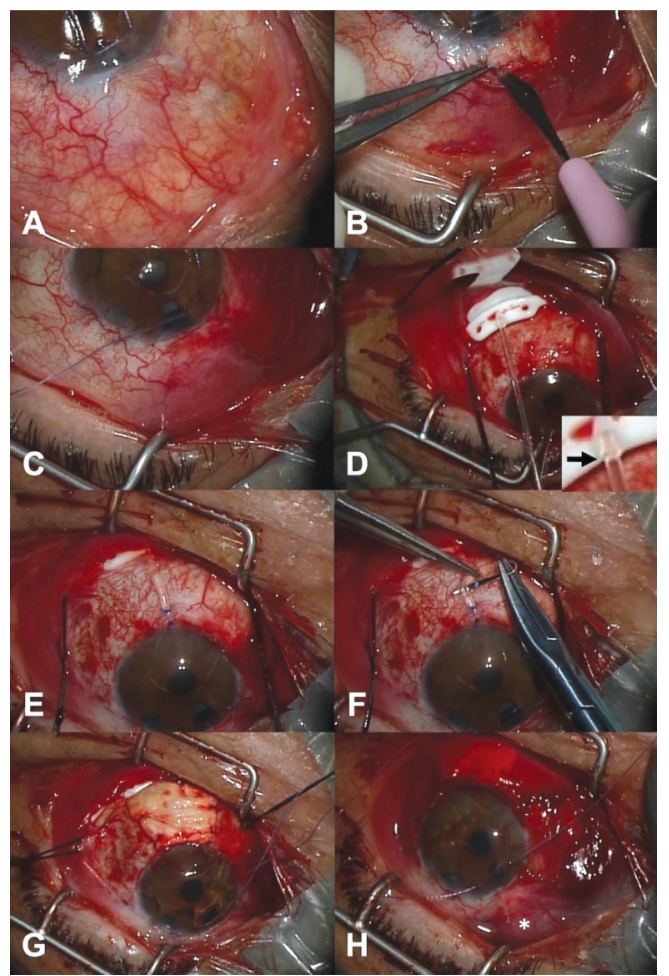

Figure 2 Patient I surgical procedure.

Notes: (A) A clear corneal traction suture is created with a 6-0 polyglycolic acid (PGA) absorbable violet braided suture (Mani Inc, Tochigi, Japan). (B) The scleral flap is dissected until the aqueous humor egresses. (C) The fornix-based conjunctival flap is closed with 10-0 nylon sutures. (D) A 350- $\mathrm{mm}^{2}$ Baerveldt glaucoma implant is positioned. The tube is tightly occluded using an 8-0 PGA absorbable violet braided suture (Mani Inc) ligature (arrow). (E) The tube is inserted into the anterior chamber. (F) Tube fenestrations are created. (G) A scleral graft is used to cover the tube. (H) *bleb formation is observed at the end of the surgery.

are well controlled by laser suture lysis, ${ }^{8}$ its effect may be uncertain because of the potential for hypotony when laser suture lysis is performed before sufficient capsular fibrosis develops around the external plate.

In patient 1 , the early postoperative IOP was well controlled due to the well-functioning bleb. The bleb promptly elevated in response to ocular massage during that period. The IOP slightly increased to $15 \mathrm{mmHg}$ on postoperative day 49 and then decreased. Given the changes in the postoperative IOP levels, we speculated that the ligature around the tube dissolved and aqueous drainage began at about that time.

In patient 2 , the postoperative IOP was higher, and varied with poor bleb formation compared to patient 1 . Because the bleb was failing, bleb needling was performed on postoperative day 6 to restore bleb function. After that, the IOP was controlled, indicating that this technique, commonly used after trabeculectomy, was also applicable in this combined surgery. Considering the changes in IOP, which peaked on postoperative day 91, we speculated that the ligature around the tube dissolved at about that time. The slight increase in IOP in the late follow-up period might reflect the onset of 


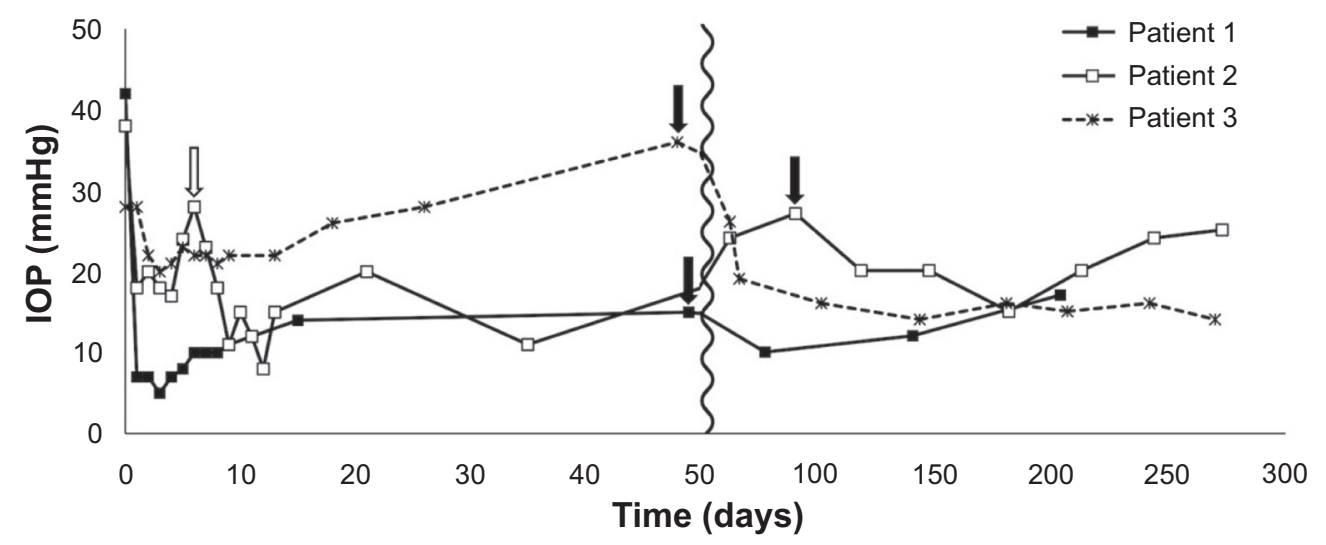

Figure 3 Changes in the postoperative intraocular pressures in the three cases.

Notes: The black arrows indicate the presumed times when the ligatures around the tubes dissolved. The white arrow indicates when bleb needling was performed in patient 2.

Abbreviation: IOP, intraocular pressure.

fibrosis around the plate, leading to subsequent IOP elevation in the late postoperative period.

Patient 3 followed what is considered to be a typical time course after BGI surgery alone. The IOP remained relatively high with full topical antiglaucoma medications, indicating that the tube fenestrations were partially effective for lowering IOP, but insufficient for reducing the IOP to a safe level. Considering the changes in IOP, we speculated that the ligature dissolved at about postoperative day 48 .

Bleb prognosis might be poor because these patients are at high risk for bleb failure due to excessive wound healing processes. ${ }^{9,10}$ Our results suggested that 7 to 13 weeks is adequate to surpass the hypertensive phase. Therefore, we performed surgical bleb revision without intraoperative application of mitomycin $\mathrm{C}$ because of concerns about toxicity. ${ }^{11}$ However, this combined surgery is time-consuming and new complications related to surgical bleb revision can occur. Further studies with more patients are needed to determine the efficacy of the combined surgery.

In conclusion, we described the combined BGI surgery and surgical bleb revision. The procedure might prevent the hypertensive phase that can occur after BGI surgery.

\section{Disclosure}

The authors declare no conflicts of interest in this paper.

\section{References}

1. Molteno AC, Polkinghorne PJ, Bowbyes JA. The vicryl tie technique for inserting a draining implant in the treatment of secondary glaucoma. Aust N Z J Ophthalmol. 1986;14(4):343-354.

2. Gedde SJ, Herndon LW, Brandt JD, Budenz DL, Feuer WJ, Schiffman JC. Postoperative complications in the Tube Versus Trabeculectomy (TVT) study during five years of follow-up. Am J Ophthalmol. 2012;153(5):804-814.

3. Shirato S, Suzuki Y, Tanihara H, Chihara E, Fuse N. Guidelines for glaucoma tube-shunt surgery. Nihon Ganka Gakkai Zasshi. 2012; 116(4):388-393. Japanese.

4. Sherwood MB, Smith MF. Prevention of early hypotony associated with Molteno implants by a new occluding stent technique. Ophthalmology. 1993;100(1):85-90.

5. Gedde SJ, Schiffman JC, Feuer WJ, Herndon LW, Brandt JD, Budenz DL. Treatment outcomes in the Tube Versus Trabeculectomy (TVT) study after five years of follow-up. Am J Ophthalmol. 2012;153(5):789-803.

6. Ayyala RS, Zurakowski D, Smith JA, et al. A clinical study of the Ahmed glaucoma valve implant in advanced glaucoma. Ophthalmology. 1998;105(10):1968-1976.

7. Yablonski ME. Trabeculectomy with internal tube shunt: a novel glaucoma surgery. J Glaucoma. 2005;14(2):91-97.

8. Trible JR, Brown DB. Occlusive ligature and standardized fenestration of a Baerveldt tube with and without antimetabolites for early postoperative intraocular pressure control. Ophthalmology. 1998;105(12):2243-2250.

9. Skuta GL, Parrish RK, 2nd. Wound healing in glaucoma filtering surgery. Surv Ophthalmol. 1987;32(3):149-170.

10. Broadway DC, Grierson I, Hitchings RA. Local effects of previous conjunctival incisional surgery and the subsequent outcome of filtration surgery. Am J Ophthalmol. 1998;125(6):805-818.

11. Derick RJ, Pasquale L, Quigley HA, Jampel H. Potential toxicity of mitomycin C. Arch Ophthalmol. 1991;109(12):1635.
Clinical Ophthalmology

\section{Publish your work in this journal}

Clinical Ophthalmology is an international, peer-reviewed journal covering all subspecialties within ophthalmology. Key topics include: Optometry; Visual science; Pharmacology and drug therapy in eye diseases; Basic Sciences; Primary and Secondary eye care; Patient Safety and Quality of Care Improvements. This journal is indexed on Submit your manuscript here: http://www.dovepress.com/clinical-ophthalmology-journal

\section{Dovepress}

PubMed Central and CAS, and is the official journal of The Society of Clinical Ophthalmology (SCO). The manuscript management system is completely online and includes a very quick and fair peer-review system, which is all easy to use. Visit http://www.dovepress.com/ testimonials.php to read real quotes from published authors. 